\title{
Paraplegia due to a ruptured aneurysm of the distal posterior inferior cerebellar artery
}

\author{
Shiro Kashiwagi, Eiji Tsuchida, Yujiro Shiroyama, Haruhide Ito, Tetsuo Yamashita
}

\begin{abstract}
A case of paraplegia was due to a ruptured aneurysm of the distal posterior inferior cerebellar artery. The paraplegia was caused by a unilateral lesion located between the cervicomedullary junction and the $\mathrm{C} 2$ level, where it involved both crossed and uncrossed pyramidal fibres projecting to the lower extremities. Since a vascular lesion near the cervicomedullary junction is likely to be missed, special attention should be paid to this region when investigating subarachnoid haemorrhage with paraplegia.
\end{abstract}

(F Neurol Neurosurg Psychiatry 1992;55:836-837)

Paraplegia is usually a sign of bilateral involvement of the corticospinal tract in the thoracic or lumbar region, or the medial motor cortex in the interhemispheric fissure. When seen in patients with subarachnoid haemorrhage (SAH), it is most probably due to rupture of an aneurysm or arteriovenous malformation (AVM) in the distribution of the anterior cerebral artery causing mechanical compression by a haematoma in the interhemispheric cistern or ischaemia resulting from vasospasm of the anterior cerebral arteries.

Our patient with SAH presented with paraplegia due to a ruptured aneurysm of the distal posterior inferior cerebellar artery (PICA).

\section{Case report}

The patient, a 57 year old woman, had been well until the morning of 23 December 1990, when she was found lying unconscious on the street. She was taken to a local hospital. On arrival at the emergency room, she was semicomatose with shallow, irregular breathing. The pupils were miotic, and there was transient skew deviation. She was able to move the upper limbs, better on the left side, but did not move the lower limbs in response to painful stimuli. With respiratory support by bag and face mask, her breathing improved and she began to open her eyes in response to pain. CT scan showed diffuse subarachnoid haemorrhage in both the supra- and infratentorial regions with haemorrhage in the fourth ventricle and the posterior horn of the left lateral ventricle. She was transferred to our hospital.

On admission, her blood pressure was $140 / 90$, pulse rate $92 / \mathrm{min}$, respiration rate
$36 / \mathrm{min}$, and body temperature $37 \cdot 6^{\circ} \mathrm{C}$. She opened her eyes in response to pain. The pupils were isocoric and responded to light. She moved the upper limbs and was able to localise pain on both sides, better on the left, but did not move the lower extremities in response to painful stimuli. Deep tendon reflexes were ++ in the upper limbs and + in the lower extremities. The plantar responses were extensor.

The three vessel angiography on 23 December failed to reveal any aneurysm or other vascular lesions that might be the source of the subarachnoid haemorrhage. She continued to be stuporous with paraparesis over the following three weeks, and gradually improved in the fourth week. The strength of the lower limbs had recovered to $4 / 5$ on the right and $5 / 5$ on the left by the end of the sixth week after the attack, at which time sensory examination revealed normal response to pain, touch and vibration in the upper and lower limbs. No lower cranial nerve palsy was noted. Reflexes were +2 in the upper and +3 in the lower extremities.

MRI with a 1.5-T superconducting coil was carried out on 22 January 1991, and showed a vascular lesion in the subarachnoid space adjacent to the spinal cord at the level of $\mathrm{Cl}_{1}$ (fig A). Cerebral angiography was repeated, and right vertebral angiograms revealed an aneurysm in the anterior medullary segment of the right posterior inferior cerebellar artery (PICA) (fig B).

On 18 February a right suboccipital craniotomy and $\mathrm{C} 1$ laminectomy was performed. A saccular aneurysm, $2.5 \times 6.0 \mathrm{~mm}$, arose from the anterior medullary segment of the PICA. The dome of the aneurysm was located antero-lateral to the spinal cord just rostral to the $\mathrm{C} 1$ roots. There was yellowish pigmentation on the surface of the spinal cord, suggesting previous haemorrhage. The neck of the aneurysm was clipped with a slightly curved aneurysm clip. The patient did well postoperatively. She regained the ability to walk, and was discharged from hospital on 11 April 1991. She returned to work three months later.

\section{Discussion}

Distal PICA aneurysm is rare, accounting for less than $0.5 \%$ of all intracranial aneurysms. ${ }^{1-9}$ There are no specific neurological deficits associated with rupture of distal PICA 

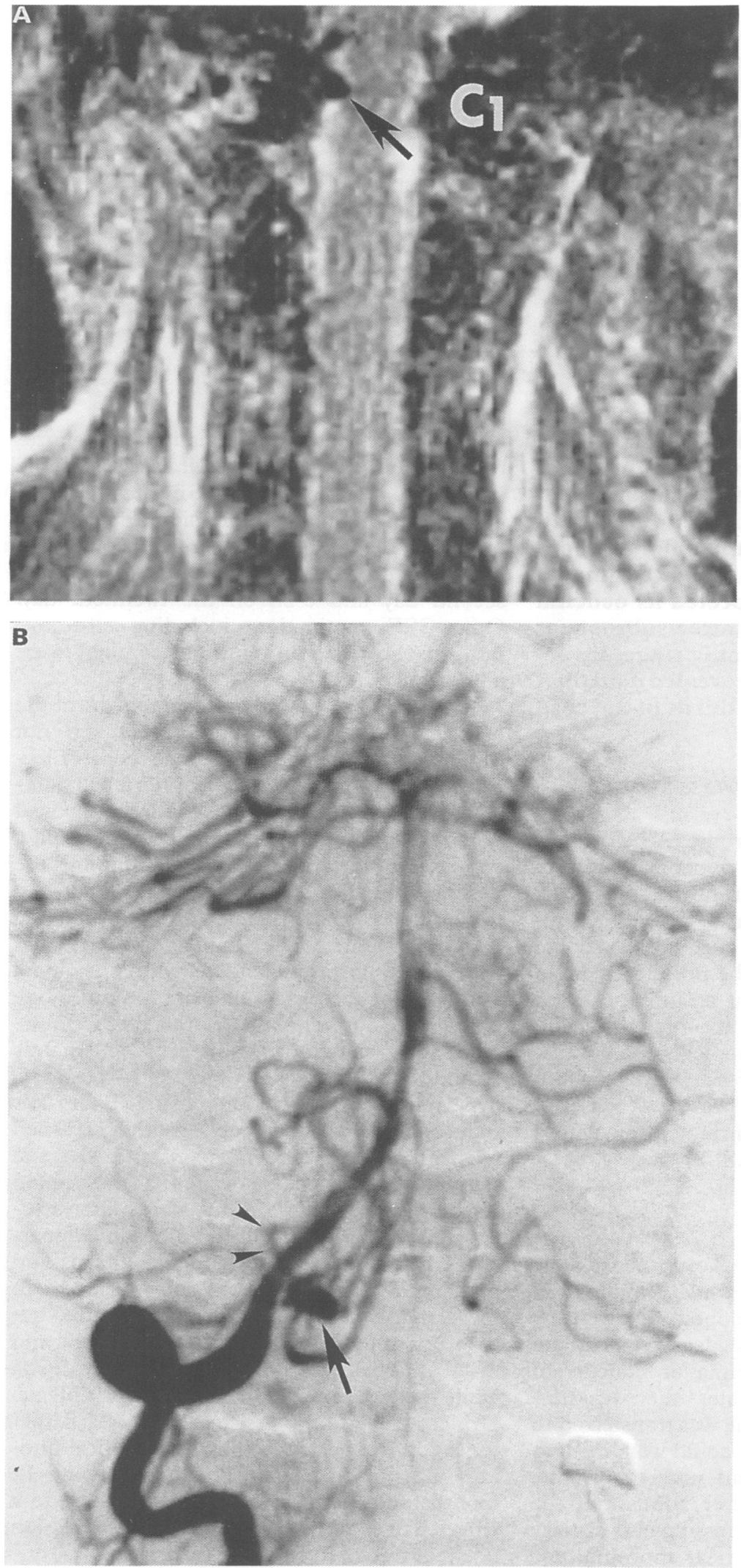

Figure A) Coronal $T_{2}$ weighted $M R I$ showing an abnormal signal-void adjacent to the spinal cord at the level of $C-1$ on the right side (arrow); B) Anterior-posterior view of right vertebral angiogram showing an aneurysm (arrow) in the anterior medullary segment of the posterior inferior cerebellar artery (arrowhead).

aneurysms. ${ }^{7}$ Dysesthesia or monoparesis of the lower limb has been described in three of 21 PICA aneurysms, ${ }^{4}$ but paraplegia has not been documented as a presenting sign of ruptured distal PICA aneurysms.

Anatomically, the pyramidal decussation extends longitudinally over from the medullocervical junction to the $\mathrm{C} 2$ level. The motor tract of the lower extremities crosses dorsal and caudal to the fibres supplying the upper limbs. The fibres projecting to the lower limbs lie laterally and dorsally, decussate between the $\mathrm{C} 1$ and C2 levels, and are again located laterally in the characteristic lamination of the lateral corticospinal tract at the $\mathrm{C} 2$ level. ${ }^{10}$ Paraplegia can be caused by a unilateral lesion between the cervicomedullary junction and C2 level, where it involves both the crossed and uncrossed pyramidal fibres projecting to the lower limbs. Since these crossed and uncrossed fibres are located laterally to those of the upper limbs, lateral compression is likely to impair the fibres projecting to the lower extremities in this particular location. A ruptured aneurysm arising from one of the medullary segments of the PICA, which accounts for approximately $50 \%$ of all distal PICA aneurysms, ${ }^{28}$ can involve both the crossed and uncrossed pyramidal fibres projecting to the lower limbs, as was seen in our case.

In our patient, MRI showed that the aneurysm was located laterally to the spinal cord at the level of $\mathrm{Cl}$, and this was confirmed at surgery. We think that the transient paraplegia was caused by mechanical compression due to local haematoma, and/or local circulatory disturbance, which impaired the more laterally located crossed and uncrossed fibres projecting to the lower extremities. Thus the paraplegia almost completely resolved after six weeks, after the disappearance of the haematoma and normalisation of the local circulation.

Distal PICA aneurysms are likely to be missed, and therefore careful investigation is necessary using 4-vessel angiography. A unilateral injection that shows reflux into the contralateral proximal PICA would be insufficient to rule out lesions more distal to the artery. ${ }^{6}$ Our case suggests that a vascular lesion in the cervicomedullary junction should be included in the differential diagnosis of paraplegia associated with SAH, and that special attention should be paid to this region in the investigation.

1 Dernbach PD, Sila CA, Little JR. Giant and multiple aneurysms of the distal posterior inferior cerebellar artery. Neurosurgery 1988;22:309-12.

2 Gács G, Viñuela F, Fox AJ, Drake CG. Peripheral aneurysm of the cerebellar arteries. Report of 16 cases. $\mathscr{f}$ Neurosur 1983;58:63-8.

3 Hiscott P, Crockard A. Multiple aneurysms of the distal posterior inferior cerebellar artery. Neurosurgery 1982; 10:101-2.

4 Hudgins RJ, Day AL, Quisling RG, Rhoton AL, Sypert GW, Bengochea FG. Aneurysms of the posterior inferior cerebellar artery. A clinical and anatomical analysis. $f$ Neurosurg 1983;58:381-7.

5 Ishikawa T, Suzuki A, Yasui N. Distal posterior inferior cerebellar aneurysms. Report of 12 cases. Neurol Med Chir cerebellar aneurysm

6 Rothman SLG, Azarkia, Kier EL, Schechter MM, Allen WE. The angiography of posterior inferior cerebellar artery aneurysm. Neuroradiology 1973;6:1-7.

7 Nishizaki T, Tamaki N, Nishida Y, Fujita K, Matsumoto S. Aneurysms of the distal posterior inferior cerebellar artery: Experience with three cases and review of the literature. Neurosurgery 1985;16:829-32.

8 Yamamoto I, Tsugane R, Ohya M, Sato O, Ogura K, Hara $M$. Peripheral aneurysms of the posterior inferior cerebellar artery. Neurosurg 1984;15:839-45.

9 Yasargil MG. Microneurosurgery, vol II. Stuttgart; Georg Thieme, 1984:290-1.

10 DeJong RN. The neurologic examination, incorporating the fundamentals of neuroanatomy and neurophysiology, 2 nd ed. New York; Hoeber-Harper, 1958:1076. 